\title{
EFFECTS OF LATE MERGERS
}

\author{
On Stellar Populations in E and SO Galaxies
}

\author{
FRANCOIS SCHWEIZER \\ Carnegie Institution of Washington, Department of \\ Terrestrial Magnetism, Washington, DC 20015, USA \\ (schweizer@bmrt.ciw.edu)
}

Perhaps the single most important component missing from Baade's famous stellar-populations paper is the cold gas, in both its atomic and molecular forms. This review of the effects of late mergers concentrates on the role that this gas plays in forming new stellar populations and defining the characteristics of merger remnants and of some early-type galaxies.

By 'late mergers' I mean those occurring during the second half of the Hubble age (last $7 \mathrm{Gyr}$ ), or at redshifts of $z \lesssim 0.5$ for $H_{0}=50$ and $q_{0}=1 / 2$. In a simple evolutionary model with exponentially declining star formation rates for disk galaxies (Searle et al., 1973), Sc galaxies 7 Gyr ago had 1/2 of their original gas left, while now they have $1 / 4$; and $\mathrm{Sb}$ galaxies then had $1 / 3$ left, while now they have $1 / 10$ th.

Recent progress in understanding effects of late mergers on stellar populations in $\mathrm{E}+\mathrm{S} 0$ galaxies has been achieved from two directions. First, the study of ongoing mergers and starbursts has made possible the prediction of population properties in remnants for comparison with early-type galaxies. Second, the study of fine structure in such galaxies has revealed correlations with colors and spectral properties. These correlations break the age-metallicity degeneracy and have permitted some first age dating of $\mathrm{E}$ and S0 galaxies. Recent results in these two areas are as follows.

\section{Galaxy Building: Mergers and Starbursts}

What happens to pre-existing, older stars when two disk galaxies of nearly equal mass merge? Obviously, the stellar contents of the two participants mix, and violent relaxation tends to redistribute them into the characteristic $r^{1 / 4}$ distribution (Toomre, 1977; Barnes, 1988). The population mixing is of no great consequence if the merging galaxies are of the same Hubble type, but is more significant if their types and chemical compositions differ, e.g., if a metal-rich S0 galaxy merges with a metal-poor Sc. 
N-body simulations by Barnes (1992) emphasize that the violent relaxation is surprisingly incomplete. Therefore, the shapes and kinematics of merger remnants are related to the merger geometry, i.e., to the relative spins of the galaxies and orbit. Different mergers leave different fractions of stars in box, X-tube, and Z-tube orbits, whence the orbit structure of remnants can yield valuable clues about past mergers (Fig. 13 in Barnes, 1994). The latest wrinkle in this story is that even small amounts of gas (1.5\% of total mass) can change this orbit structure dramatically and can lessen the kinematic-axis misalignment problems that one runs into when simulating purely stellar mergers (Barnes and Hernquist, 1992, 1995).

Gas produces the most spectacular effects during mergers. Follow-up observations to IRAS have turned up ever more luminous central starbursts, revealing the important role of gaseous dissipation. Yet, the emphasis on highly centrally concentrated starbursts has left us with two puzzles: Why are there few, if any, remnants and $\mathrm{E}+\mathrm{S} 0$ galaxies with centers bluer than their envelopes? And why do merger remnants tend to show extended A-type spectra, sometimes throughout their bodies (Schweizer, 1990)?

We have only recently come to understand that merger-induced starbursts last much longer than previously thought. Spectroscopic studies of colliding galaxies emphasize that after a first encounter, and long before the final merger, major starbursts do already occur (Bernlöhr, 1993). As the example of NGC 7714/15 suggests, by the time these two galaxies merge there will be at least two, if not three aging starburst populations, each with its distinct age and chemistry. In NGC 4038/39, a spectacular pair of merging disks, giant $\mathrm{H}$ II regions throughout these disks trace two global starbursts (Rubin et al., 1970). Recent $H S T$ observations reveal $\sim 700$ young star clusters, many associated with the H II regions and as compact as globular clusters (Whitmore and Schweizer, 1995). Thus, after violent relaxation will have run its incomplete course the merger remnant will feature a mix of aging starburst populations distributed throughout its body. This phenomenon may explain the radial near-constancy of $\mathrm{H} \beta$ absorption observed in E's and the inferred absence of age gradients (Davies et al., 1993).

Model simulations that include gas and star formation support this notion of prolonged global starbursts (Mihos et al., 1993). Specifically, after the last pericenter passage in NGC 4038/39, relatively intense star formation (5-7 $\left.M_{\odot} / \mathrm{yr}\right)$ occurs for about $600 \mathrm{Myr}$. These simulations suggest that the star-formation histories of disk-disk mergers can differ dramatically, often lasting much longer than gas-exhaustion times inferred from peak bursts would suggest. Some of these mergers will reach super-starburst status $\left(\gtrsim 50-100 M_{\odot} / y r\right)$ while others, like the Antennae, will not.

The building and twirling of dense cores in merger remnants is also 
becoming better understood. Some mergers feature central starbursts with 10-50 times the $\mathrm{H}_{2}$ content of the Milky Way (Young and Scoville, 1991). Observed central gas densities reach $\sim 500 M_{\odot} \mathrm{pc}^{-3}$ (Solomon et al., 1992) and are still limited by interferometric resolution. Such densities lie well in the range of central stellar densities in giant ellipticals. Thus, if most of this gas turns into stars, merger-induced dissipation seems capable of producing the high densities observed in cores of E's (Kormendy and Sanders, 1992).

Piled-up gas may also explain the often odd kinematics of these cores (Bender, 1990). VLA observations of the merger remnant NGC 7252 reveal that $\mathrm{H} \mathrm{I}$ from the tidal tails is falling back into the main body (Hibbard et $a l ., 1994)$ at a rate of $1-2 M_{\odot} / y r$ (Hibbard and Mihos, 1995). This infall rate, which was larger in the past, is about equal to the star formation rate in the central, oddly rotating molecular-gas disk (Wang et al., 1992). The beautiful spiral structure of this disk revealed by HST (Whitmore et al., 1993) requires a continuous source of excitation, and gaseous infall is the most likely such source (Toomre, 1990). This suggests that tail gas falling back during the past $\sim 1$ Gyr has built up the oddly rotating core of this future elliptical. Supporting this hypothesis, HST observations of cores in E's often show gas and dust disks, some blue and with hints of spiral structure (Kormendy et al., 1994; Grillmair et al., 1994). The formation of such cores through prolonged infall of tail gas in cold, directed streams may also explain the enhanced $\mathrm{Mg}$ abundances found within the core boundaries by Bender and Surma (1992). The sustained star formation induced in the central gas disk can only have fostered metal enrichment.

The formation of globular clusters (GC) from crashing gas masses during disk-disk mergers is a new research subject of considerable interest. Half a dozen galaxies are now known in which young GC systems seem to have formed in this manner (NGC 1140, 1275, 1316, 3597, 4038/39, and 7252). Although questions concerning the behavior of the specific GC frequency $S$ during mergers remain (Harris, 1995), objections against a merger formation of E's based on the presumed constancy of $S$ (van den Bergh, 1990) have been weakened: The number of GC's is not conserved, it clearly increases. Unlike Harris, I believe it is too early to tell whether non-clustered stars increase in the same proportion as clusters. This proportion may be a strong function of gas fraction and metallicity.

We are only beginning to glimpse what some of the parameters determining $S$ may be. For instance, the merger remnant NGC 3921 seems to have produced fewer very luminous GC's than its close kin NGC 7252 (unpublished). NGC 3921 was an S0-Sc merger (Hibbard and van Gorkom, 1995), while NGC 7252 was an Sc-Sc merger. Hence, the lack of two gaseous disks crashing together in NGC 3921 may have led to less, or less luminous, GC formation. Here again, the Hubble types of merger components may 
determine the kinds of new stellar populations formed in the remnants.

\section{Merger Remnants: Fine Structure and Age Dating}

The discovery of various kinds of fine structure in $\mathrm{E}$ and $\mathrm{S} 0$ galaxies during the past 15 years has opened new avenues to identifying merger remnants among these galaxies. Such fine structure, which includes ripples ("shells"), plumes, boxiness, and 'X-structure,' has been found in $\sim^{2 / 3}$ of field ellipticals and $1 / 2$ of field S0's (Table 1 in Schweizer, 1993), suggesting that many such galaxies may have experienced at least one late merger.

Were these mergers mostly minor accretions of dwarf galaxies by preexisting E and S0's (Hernquist and Quinn, 1988, 1989; Weil and Hernquist, 1993) or were they major mergers of near-equal disk galaxies (Barnes, 1988, 1992; Hernquist and Spergel, 1992)? The balance between these two possibilities is tipping increasingly in favor of major disk mergers. Insufficient numbers of dwarfs and uncomfortably large luminosities of some ripple systems argue against minor accretions, while associations between tidal tails and ripples support major mergers. The prediction (Barnes, 1992) and observational confirmation (Hibbard et al., 1994) of prolonged infall of tail material now point strongly toward major mergers: Clumpy streams of stars flung out during a merger will return for several Gyr, acting like a series of minor accretions. In this interpretation, fine structure in $\mathrm{E}+\mathrm{S} 0$ galaxies is the sum of tell-tale signatures remaining from major, structuredefining mergers.

This interpretation has been strengthened by the discoveries of correlations between fine-structure content and absorption-line strengths of $\mathrm{H} \beta$, $\mathrm{Mg}$, and CN (Carter et al., 1988; Schweizer et al., 1990) as well as UBV colors (Schweizer and Seitzer, $1992=$ SS92). These correlations hint at aging global starbursts in E + S0 galaxies with much fine structure and, thus, at major mergers. They also break the age-metallicity degeneracy in favor of age variations. Two-burst models of population evolution (based on cluster-evolution models by Bruzual and Charlot, 1993; see also Fritze-von Alvensleben and Gerhard, 1994a,b) have permitted a first age-dating of ancient mergers in 69 early-type galaxies and the identification of a number of dynamically young E's (SS92). The latter's youth is now also supported by a more thorough, though only single-burst, population synthesis (González, 1993; Faber, 1995). According to the two-burst models the scatter of $U B V$ colors suggests that in the field $\mathrm{E}+\mathrm{S} 0$ formation via major mergers occurred for at least 5-10 Gyr, or about $1 / 3-2 / 3$ the age of the universe. 


\section{Conclusions}

Late mergers and gas appear to have played a major role in determining the kinematics and chemical composition of stellar populations in many early-type galaxies. Both the merger geometry and the Hubble types of component galaxies have imprinted the remnant populations. In general, merger-induced starbursts are likely to have been prolonged $(0.2-1 \mathrm{Gyr})$ and multi-peaked, leading to the formation of subpopulations (stars, GC's) with different spatial, kinematic, and chemical distributions. The formation of present-day E and S0 galaxies seems to have occurred for at least 510 Gyr: Aging starbursts now reveal themselves through increased color and line-strength scatter. This and other evidence suggests that Hubble's morphological sequence may rank galaxies mainly by the number, type, and vehemence of mergers in their past history.

I thank J. Barnes, J. Hibbard and B. Whitmore for their kind permission to cite results in advance of publication, and I gratefully acknowledge partial support through an IAU travel grant and NSF grant AST 92-21423.

\section{References}

Barnes, J.E., 1988, Ap. J. 331, 699

Barnes, J.E., 1992, Ap. J. 393, 484

Barnes, J.E., 1994, The Formation of Galaxies, Cambridge Univ., Cambridge, in press

Barnes, J.E. and Hernquist, L.E., 1992, Ann. Rev. Astron. Astroph. 30, 705

Barnes, J.E. and Hernquist, L.E., 1995, in preparation

Bender, R., 1990, Dynamics and Interactions of Galaxies, Springer, Heidelberg, p. 232

Bender, R. and Surma, P., 1992, A. \& A. 258, 250

Bernlöhr, K., 1993, A. \& A. 268, 25

Bruzual, A.G. and Charlot, S., 1993, Ap. J. 405, 538

Carter, D. et al., 1988, M. N.R. A. S. 235, 813

Davies, R.L., Sadler, E.M. and Peletier, R.F., 1993, M. N. R. A. S. 262, 650

Faber, S.M., 1995, this volume

Fritze-von Alvensleben, U. and Gerhard, O.E., 1994a, $A$. $\& A$ 285, 751

Fritze-von Alvensleben, U. and Gerhard, O.E., 1994b, $A$. $\mathscr{E} A .285,775$

González, J.J., 1993, Ph.D. thesis, UC Santa Cruz

Grillmair, C.J. et al., 1994, A. J. 108, 102

Harris, W.E., 1995, this volume

Hernquist, L. and Quinn, P.J., 1988, Ap. J. 331, 682

Hernquist, L. and Quinn, P.J., 1989, Ap. J. 342, 1

Hernquist, L. and Spergel, D.N., 1992, Ap. J. 399, L117

Hibbard, J.E., Guhathakurta, P., van Gorkom, J.H. and Schweizer, F., 1994, A. J. 107, 67

Hibbard, J.E. and Mihos, J.C., 1995, preprint

Hibbard, J.E. and van Gorkom, J.H., 1995, in preparation

Kormendy, J. and Sanders, D.B., 1992, Ap. J. 390, L53

Kormendy, J. et al., 1994, Workshop on Dwarf Galaxies, ESO, Garching, in press

Mihos, J.C., Bothun, G.D. and Richstone, D.O., 1993, Ap. J. 418, 82

Rubin, V.C., Ford, W.K. and D'Odorico, S., 1970, Ap. J. 160, 801

Schweizer, F., 1990, Dynamics and Interactions of Galaxies, Springer, Heidelberg, p.60

Schweizer, F., 1993, Structure, Dyn. and Chem. Evol. of E Gal., ESO, Garching, p.651 
Schweizer, F. et al., 1990, Ap. J. 364, L33

Schweizer, F. and Seitzer, P., 1992, A. J. 104, 1039 (SS92)

Searle, L., Sargent, W.L.W. and Bagnuolo, W.G., 1973, Ap. J., 179, 427

Solomon, P.M., Downes, D. and Radford, S.J.E., 1992, Ap. J. 387, L55

Toomre, A., 1977, Evolution of Galaxies and Stellar Populations, Yale, New Haven, p.401 Toomre, A., 1990, Dynamics and Interactions of Galaxies, Springer, Heidelberg, p.292 van den Bergh, S., 1990, Dyn. and Interactions of Galaxies, Springer, Heidelberg, p.492 Wang, Z., Schweizer, F. and Scoville, N.Z., 1992, Ap. J. 396, 510

Weil, M.L. and Hernquist, L., 1993, Ap. J. 405, 142

Whitmore, B.C. et al., 1993, A. J. 106, 1354

Whitmore, B.C. and Schweizer, F., 1995, A. J., submitted

Young, J.S. and Scoville, N.Z., 1991, Ann. Rev. Astron. Astroph. 29, 581

VAN DEN BERGH: I was delighted to see the beautiful luminosity function that you and Whitmore have obtained with $H S T$ for the clusters in 'The Antennae.' The fact that the luminosity function continues to rise over a factor of $\sim 100$ in luminosity clearly shows that these are open clusters, rather than globulars which have a narrow Gaussian luminosity function. In other words, mergers seem to produce open clusters, not globulars.

SCHWEIZER: Categories of astronomical objects are generally defined by their objects' physical characteristics, rather than by their luminosity function (LF). The latter is derived after the object category has been defined. Specifically, a narrow Gaussian LF seems to be a property of old GC's, but we don't know the LF of young GC's because we do not yet fully understand cluster destruction mechanisms. It could be that the LF of clusters in 'The Antennae' refers to a mixture of globular and open clusters.

BALCELLS: The simple, clean geometry of the NGC 7252 system, with the expelled gas lying along thin filaments, has provided us with a clean example of what should be a general phenomenon in mergers of gas-rich systems; tidal debris are expelled with a range of binding energies, from bound to unbound, hence some material is bound to return (in the absence of subsequent traumas such as evaporation to a hot phase). Thus, during an interaction the galaxy "fills up its refrigerator" with fresh gas, which then slowly returns to the main body. The interaction may thus affect the star formation in the galaxy over a very long time span. The storage of gas at high galactocentric distances could be quite common. For example, minor mergers can pump up gas efficiently for some encounter geometries.

SCHWEIZER: The latter is news to me. I would have thought that only major disk-disk mergers were capable of ejecting several $10^{9} M_{\odot}$ of HI. 